\title{
Digital Workflow Used to Fabricate Implant-Supported Three-Unit Fixed Dental Prosthesis
}

\author{
Christiaan WP Pol ${ }^{1}$, DDS / Gerry M Raghoebar, MD, $\mathrm{PhD}^{2}$ / Wouter Kerdijk, $\mathrm{PhD}^{3}$ / \\ Marco S Cune DDS, PhD ${ }^{1}$ / Henny JA Meijer, DDS, PhD ${ }^{1,2}$
}
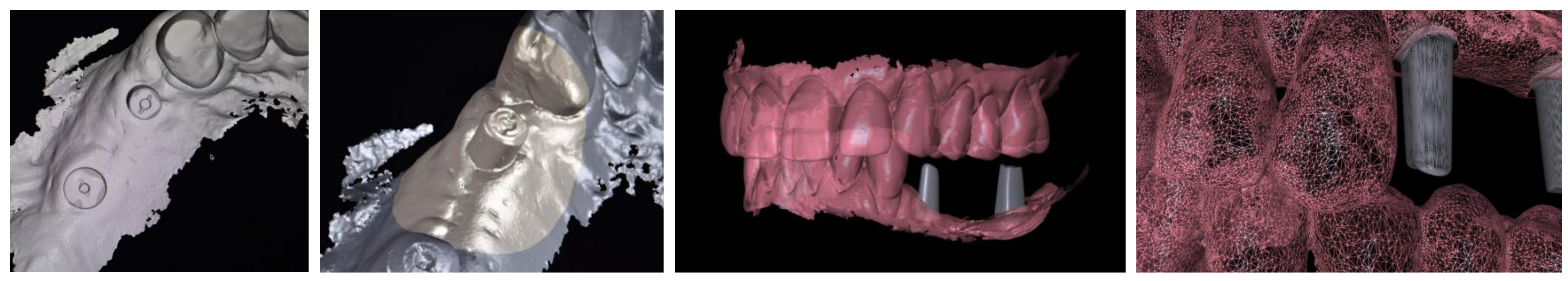

\section{Background}

The use of chair-side oral scanning for multi-unit implant prostheses is still limited, even though an increasing number of dentists are incorporating computer aided design and manufacturing $(\mathrm{CAD} / \mathrm{CAM})$ in everyday practice, and the potential for saving precious time and money is enormous. There are coded healing abutments available that allow for intra-oral scanning and a digital prosthetic workflow without first replacing the healing abutments by scanning abutments.

\section{Aim/Hypothesis}

By conducting a study on CAD/CAM 3-unit prostheses, the current state of the digital workflow and the accompanying pitfalls and possibilities are explored, with the expectation that the use of an oral scanner would allow for the fabrication of abutments and bridges with good clinical results.
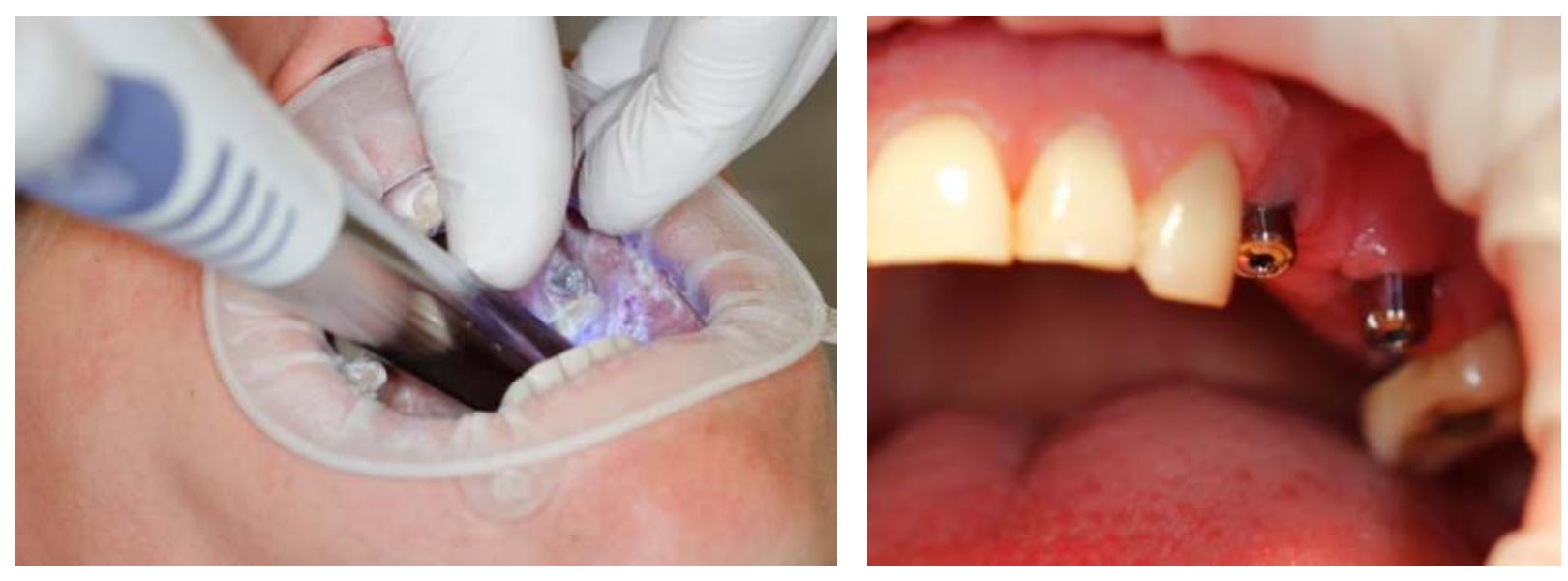

\section{Material and Methods}

In an IRB approved prospective trial, in healthy patients two dental implants (4mm width, length $8.5-15 \mathrm{~mm}$ ) were inserted to replace three missing posterior teeth. After osseointegration, a coded healing abutment was placed, and subsequently fullarch intra-oral scans were made to produce patient specific titanium abutments and a full-zirconia bridge. Clinical variables were measured before treatment, after loading and at one year after loading. Radiographic bone height was measured on radiographs of time of loading and one year after. Patient satisfaction was measured using questionnaires, complications were noted.

University Medical Center Groningen

\section{Results}

All patients $(n=60$, mean age 60.8 years $[35.7-78.1], 30 \mathrm{~m} / 30 \mathrm{f})$ were treated with CAD/CAM abutments and bridges $(n=60,33$ maxilla, 27 mandibula); average follow-up was 13 months (range 1-35). One implant was lost before loading. At 1-year $(\mathrm{n}=28)$, average radiographic bone loss was $0.04 \mathrm{~mm}$ and no significant changes in plaque, bleeding, probing pocket depth mucositis $(n=2)$ could be treated. Some scans had to be redone $(n=7)$ and many bridges were rejected $(n=20)$ before delivery because the color did not match. After test-fitting, adjustment of color $(n=2)$, design $(n=2)$ or occlusion $(n=2)$ was performed. Slight adjustment of contact, occlusion or articulation was common $(n=35)$ at cementation. The dentists involved, reported slight color mismatch $(43 \%)$ and detectable margin discrepancy (5\%). Patients were highly satisfied with design (4.3/5), color (4.2/5) and overall treatment result (4.9/10 before, 8.1/10 after treatment).
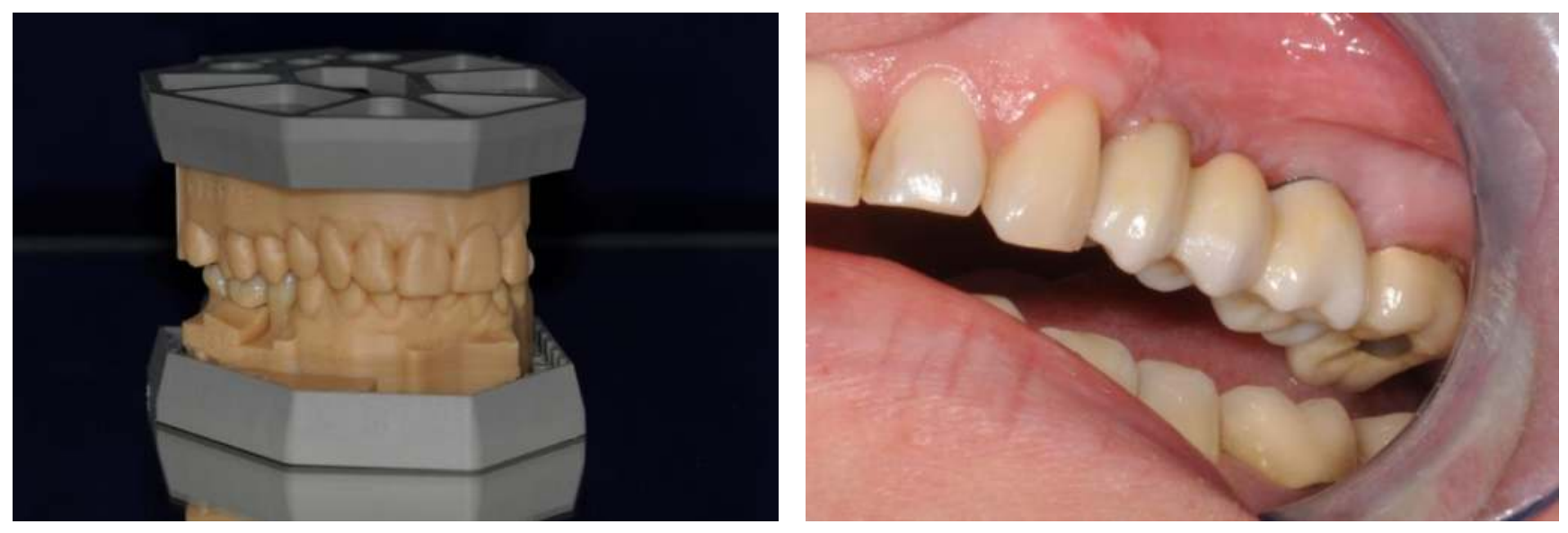

Conclusion and Clinical implications

The application of CAD/CAM showed promising results with good clinical results and high patient satisfaction, but the high number of rejections and adjustments would need to be addressed. The shortcomings we encountered in this workflow were mainly due to the laboratory procedures and the restorative materials used. After both the dentist and the dental lab have gained sufficient experience with the digital workflow, it becomes a feasible alternative to the conventional impression workflow.

1. Department of Fixed and Removable Prosthodontics and Biomaterials, Center for Dentistry and Oral Hygiene, University Medical Center Groningen, The Netherlands

2. Department of Oral and Maxillofacial Surgery, University Medical Center Groningen, The Netherlands

3. Department of Public and Individual Oral Health, Center for Dentistry and Oral Hygiene, University Medical Center Groningen, The Netherlands or gingiva index occurred. Loosening $(n=3)$ and periimplant 